\title{
A Survey on Cross Layer Distributed Topology Control in Mobile Adhoc Network
}

\author{
M.S. Brindha
}

\begin{abstract}
Mobile Ad-Hoc Networks (MANETs) have numerous restrictions because of its changing nature. So the routing and topology control algorithms for MANETs should be designed in such a way that on any instance the network should ensure minimum interference among the nodes.In this survey paper, we are going to discuss about the various methods which are used to satisfy the interference condition and delay among the nodes in the MANETs.
\end{abstract}

Keywords--- Cross Layer Protocols, Delay, MANETs, Interference.

\section{INTRODUCTION}

$\mathrm{M}$ OBILE Adhoc Network (MANET) is a set of nodes associated through a wireless medium with rapidly changing topologies. It can be set up anytime, anywhere without the need of any centralized base station. Hence find wide applications in regions where network needs to be quickly established such as disaster recovery operation, battlefield communication etc. In conventional wireless network the nodes communicate with other nodes over the path described by Base station (BS). In Adhoc networks tracking, routing and route maintenance is done solely by nodes. These nodes are portable and battery operated. Due to restricted battery resources and node mobility, multihop routes are used to cover the changing network environment. Thus a single node failure in MANET can lead to losing connectivity and network partitioning. Moreover it is very difficult or even impossible to recharge or replace the batteries of nodes once deployed in many applications. It is therefore required to limit power consumption, improve the robustness of system and prolong the battery life.

MANET (Mobile Ad-hoc Network) is a famous and widely used wireless network. MANET is a sort of selforganizing and decentralized device. It is a network made up of diverse wireless mobile nodes which collectively work together so that transmission is viable among any of the nodes in the device. Nodes communicate with each other with the direct shared wireless radio links. All the mobile hosts act as routers in the network. Due to open and dynamic nature, this network is quite prone to range of attacks. Information in the type of packets is transmitted from source to destination with the help of different nodes in the route. There are certain things which needs to be noticed as Route selection, Request initiation, topology used and so forth.

M.S. Brindha, M.E. (CSE), Sri Venkateswara College of Engineering, Pennalur, Sriperumbudur, Tamilnadu.

DOI:10.9756/BIJNTA.8346
The routing scheme in MANET is more challenging than traditional networks. To handle the network with large number of hosts, many routing protocols like AODV, DSR with limited resources like energy and bandwidth but no security consideration have been made. Further, many secure routing protocols are developed to secure the network. Trust is defined as a degree of belief among various entities. The trust for same entity can be different when evaluated by different people. Trust management is a system that will assure various important features like security, access control, intrusion detection, isolating malicious nodes etc.

\section{RELATED WORK}

Sirwen A. Mohammed et.al discussed about how to use NS2simulation for designing wireless networks and using Cryptography algorithm as to security information. It briefly describes the basic wireless networks categories, analyzes wireless LAN networks, briefly describes their components and technologies, explains the Wi-Fi technology and analyzes property sources related to wireless networks simulators and its detailed description, Specify the configuration for the simple wireless network and create corresponding model by using ns-2 simulator, demonstrates selected characteristics of the specified network configuration using the simulation model, and show scenario of transmission data among nodes[1].

Samba Sesay et.al discussed about the broad range of research issues such as Routing, Medium Access, Multicasting, Quality of service, TCP performance, Energy, Security and Bluetooth, outlining the major challenges which have to be solved before widespread deployment of the technology is possible. Through this survey it would be seen that Ad hoc Networking presence an interesting research area inheriting the problems of wireless and mobile communications in their most difficult form[2].

Ranjana Sharma et.al presented a paper in which it mainly focused upon the architecture, history, types of Attacks, comparative analysis of previous techniques already found. Also a brief introduction is given on MANET, few routing protocols, Trust and trust management with a Trust based Routing Protocol to mitigate the effect of various attacks like Black hole effect etc that occurs due to dynamic behaviour or malicious nodes with the communication process[3].

Vinit Garg et.al proposed an approach in which the ad-hoc routing protocols, the attackers, attacks by the attackers and some suggestions and solution for secure routing protocols that follow the table driven and on-demand approaches are discussed. With the advances of wireless communication technology, low-cost and powerful wireless transceivers are 
widely used in mobile applications. Mobile networks have attracted significant interests in recent years because of their improved flexibility and reduced costs. A comparison between proposed solutions can provide the basis for future research with some analysis and suggestions[4].

Marcelo G. Rubinstein et.al presented a paper in which the overview of issues related to medium access control (MAC), routing, and transport in wireless ad hoc networks and techniques proposed to improve the performance of protocols are discussed. Research activities and problems requiring further work are also presented. Finally, the paper presents a project concerning an ad hoc network to easily deploy Internet services on low-income habitations fostering digital inclusion[5].

Wenjia Li et.al proposed an approach and discussed about the security issues and their current solutions in the mobile ad hoc network. Owe to the vulnerable nature of the mobile ad hoc network, there are numerous security threats that disturb the development of it. We first analyze the main vulnerabilities in the mobile ad hoc networks, which have made it much easier to suffer from attacks than the traditional wired network. Then we discuss the security criteria of the mobile ad hoc network and present the main attack types that exist in it. Finally, we survey the current security solutions for the mobile ad hoc network[6].

\section{ALGORITHMS INVOLVED IN MANET FOR TOPOLOGY CONTROL}

\section{A. Node Classification Rule}

(i) Stable Skeletal Node: A node $\mathrm{u}$ is stated to be a established skeletal node if it has two acquaintances $\mathrm{v}$ and $\mathrm{w}$ such that $\mathrm{v}$ and $\mathrm{w}$ are not neighbour of each other and $\mathrm{u}$ is the only common neighbor for both $\mathrm{v}$ and $\mathrm{w}$. In other terms, the only shortest path between $\mathrm{v}$ and $\mathrm{w}$ is the node $\mathrm{u}$ which is the mid-point. Since SG is required to provide shortest paths between every node pair and since $u$ is the only intermediate node on the shortest path between $\mathrm{v}$ and $\mathrm{w}$, $\mathrm{u}$ must be included in any SG that is an mCDS. (ii) Stable Non-Skeletal Node: Node $\mathrm{x}$ is said to be a stable non-skeletal node if, for each pair of neighbors $\mathrm{y}$ and $\mathrm{z}$ of node $\mathrm{x}$, nodes $\mathrm{y}$ and $\mathrm{z}$ are either (1) neighbors of each other (i.e) directly connected or (2) y and $z$ are connected by a step path going through a stable skeletal node. (iii). Bi-stable Skeletal and Bistable nonSkeletal nodes. All other nodes that are not classified as either a stable skeletal node or a stable non-skeletal node are bi-stable undecided nodes. The bi-stable nodes can become skeletal nodes or nonskeletal nodes. The bi-stable nodes are in "undecided" state. We will change the state of each bi-stable node into either a skeletal node or a non-skeletal node after further information exchange. It is simple to be implemented and can be executed faster. It also has less time complexity.

\section{B. XTC Algorithm}

I) Neighbor ordering

II) Neighbor order exchange

III) Edge selection. In the first step each network node u computes a total order $\prec$ u over all its neighbors in the network graph G. From an abstract point of view, this order is intended to reflect the quality of the links to the neighbors. A node u will consider its neighbors in $\mathrm{G}$ (in the third step of the algorithm) according to $\prec \mathrm{u}$ ordered with respect to decreasing link quality: The link to a neighbor appearing early in the order $\prec u$ is regarded as being of higher quality than the link to a neighbor placed later in $<\mathrm{u}$. A neighbor $\mathrm{w}$ appearing before $\mathrm{v}$ in order $\prec \mathrm{u}$ is denoted as $\mathrm{w}<\mathrm{u} v$. For illustration, we assume in Section 4 that $\prec u$ corresponds to the order of the neighbors' Euclidean distances from u. It is, however conceivable that the neighbor order reflects a much more general notion of link quality, such as signal attenuation or packet arrival rate. In the second step the neighbor order information is exchanged among all neighbors. Typically a node u broadcasts its own neighbor order while receiving the orders established by all of its neighbors. During the third step, which does not require any further communication, each node locally selects those neighboring nodes, which will form its neighborhood in the resulting topology control graph, based on the previously exchanged neighbor order information. For this purpose a node u traverses $\prec u$ with decreasing link quality: "Good" neighbors are considered first, "worse" ones later. Informally speaking, a node u only builds a direct communication link to a neighbor v if u has no "better" neighbor w that can be reached more easily from $\mathrm{v}$ than $\mathrm{u}$ itself. The simulation results show that power consumption is better when compared to AODV routing protocol.

\section{Energy Efficiency and Minimum Delay Algorithm}

First of all shortest path is to be considered between every node pair. After finding the shortest path, the data packet is to be transferred from source to destination. When data is sent from source to destination, it may be possible that any intermediate node can be considered more than once and more power is needed for a particular node. Due to this problem energy distribution is disturbed in the whole network and we have to distribute the equal energy to the whole network. We take a counter value for network and consider a threshold value for every node in the network and check if specific node's value exceeding a given value then another node is to be considered for data packet transmission. In that way the energy consumption of every node does not exceeds to a given value and energy is equally distributed in the whole network.

\section{Hybrid Algorithm}

According to the proposed algorithm, when a node detects a clog, it will inform the source node from which it has received packets to send with the lowest data rate. To accomplish this, it transmits a back propagating message to the source. Deliberately, it has been restricted the transmission of the source node with the most minimal information rate and to continue serving the nodes with higher information rates keeping in mind the goal to keep up ideal throughput. At the point when every node in this transmission path gets the data, it checks whether it can apply Resource Control12 before the message finally reaches the originating node.It tries to increase 
the efficiency of the MANET in terms of throughput and network lifetime.

\section{E. Interference Based Control Topology Algorithm}

The purpose of this algorithm is used for delay-constrained mobile ad hoc networks. The goal of the topology control algorithm is to regulate the power transmission and also to minimize the interference, which is ambiguous to the prerequisite of delay constraint. When power transmission is improved to decrease the delay, which increases the number of neighbors covered by the transmission range and it cause more interference from other active nodes in the network. Therefore, we create a tradeoff between reducing delay and minimizing the interference.By using iteration, the power consumption is minimized and the interference restriction is also resolved. Then, the transmit power is increased to meet the delay constraint. The Interference-Based Topology Control algorithm controls the topology to satisfy the interference restriction, and increases the transmit range to meet the delay requirement. The simulation results show that ITCD can reduce the delay and improve the throughput performance effectively in delay-constrained mobile adhoc networks.

\section{CONCLUSION}

The conclusion we have drawn from our survey is with the purpose of reducing interference and delay among the nodes. The delay and interference restriction is attained by removing the unstable links among the nodes, so it leads to better Quality Of Service (QOS).

In this survey paper we discussed about the various algorithms which are used to improve the power optimization in mobile adhoc network has been presented. During the survey, we also find some points that can be further explored in the future, such as some aspects of the transmission, contention and queuing delay techniques can be further improved.

\section{REFERENCES}

[1] S.A. Mohammed, "design of wireless network based on NS2", Journal of Global Research in Computer Science, Vol. 3, No. 12, Pp.1-7, 2013.

[2] S. Sesay, Z. Yang and J. He, "A survey on mobile ad hoc wireless network”, Information Technology Journal, Vol. 3, No. 2, Pp.168-175, 2004.

[3] Ranjana Sharma and Anuradha Panjeta, "A Survey on Trust Based Mobile Ad-Hoc Networks", International Journal of Advanced Research in Computer Science and Software Engineering, Vol. 6, No. 6, Pp. 64-67, 2016.

[4] Vinit Garg, Manoj Kr. Shukla, Tanupriya Choudhury and Charu Gupta, “Advance Survey Of Mobile Ad-hoc Network”, IJCST, Vol. 2, No. 4, Pp. 552-555, 2011.

[5] M.G. Rubinstein, I.M. Moraes, M.E.M. Campista, L.H.M.Costa and O.C.M. Duarte, "A survey on wireless ad hoc networks", Mobile and Wireless Communication Networks, 2006.

[6] O.O. Obi, "Security issues in mobile ad-hoc networks: a survey", The 17 th White House Papers Graduate Research In Informatics at Sussex, 2004.

[7] R. Saveetha, R. Sayastri and S. Abraar, "A Distributed Transmission Power Control Protocol for End to End Delay”, International Journal of Science Technology and Management, Vol. 5, No. 04, Pp. 9-14, 2016.

[8] S. Venkatesan and C.D. Young, "A distributed topology control algorithm for MANETS”, Proc. of IEEE MILCOM'05, 2005.

[9] Q. Guan, F.R. Yu, S. Jiang, V.C. Leung and H. Mehrvar, "Topology control in mobile ad hoc networks with cooperative communications”, IEEE Wireless Communications, Vol. 19, No. 2, 2012.
[10] Y. Hu, D. Liu and Y. Wu, "A new distributed topology control algorithm based on optimization of delay in ad hoc networks", IEEE International Conference on Computer Communication and the Internet, Pp. 148-152, 2016. 